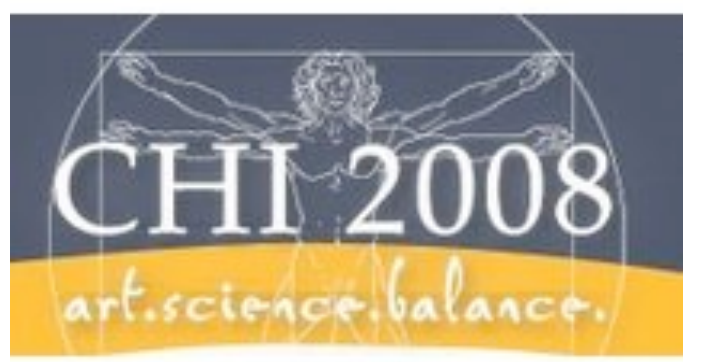

CHI 2008 Workshop Proceedings

Sunday, April $6^{\text {th }} 2008$

\title{
User Interface Description Languages for Next Generation User Interfaces
}

\author{
Orit Shaer \\ Robert J.K Jacob \\ Mark Green \\ Kris Luyten
}

\author{
Tufts University \\ Tufts University \\ University of Ontario Institute of Technology \\ Hasselt University and \\ Transnationale Universiteit Limburg
}

www.cs.tufts.edu/ oshaer/workshop/ 


\section{User Interface Description Languages for Next Generation User Interfaces}

\section{Orit Shaer}

Computer Science Department

Tufts University

Medford, MA 02155 USA

oshaer@cs.tufts.edu

Robert J.K. Jacob

Computer Science Department

Tufts University

Medford, MA 02155 USA

jacob@cs.tufts.edu

Mark Green

University of Ontario

Institute of Technology

Ontario, Canada

Mark.green@uoit.ca

\section{Kris Luyten}

Expertise Centre for Digital Media

Hasselt University and

transnationale Universiteit Limburg

Diepenbeek, Belgium

kris.luyten@uhasselt.be

Copyright is held by the author/owner(s).

CHI 2007, April 28 - May 3, 2007, San Jose, USA

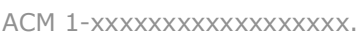

\begin{abstract}
In recent years HCI researchers have developed a broad range of new interfaces that diverge from the "window, icon, menu, pointing device" (WIMP) paradigm, employing a variety of novel interaction techniques and devices. Developers of these next generation user interfaces face challenges that are currently not addressed by state of the art user interface software tools. As part of the user interface software community's effort to address these challenges, the concept of a User Interface Description Language (UIDL), reemerge as a promising approach. To date, the UIDL research area has demonstrated extensive development, mainly targeting multi-platform and multi-modal user interfaces. However, many open questions remain regarding the usefulness and effectiveness of UIDLs in supporting the development of next generation interfaces.

The aim of this workshop is to bring together both developers of next generation user interfaces and UIDL researchers in an effort to identify key challenges facing this community, to jointly develop new approaches aimed at solving these challenges and finally to consider future spaces for UIDL research.
\end{abstract}

\section{Keywords}

User Interface Description Language (UIDL), User Interface Management System (UIMS). Next

Generation User interfaces. 


\section{ACM Classification Keywords}

H5.m. Information interfaces and presentation

\section{Motivation}

In the last decade, new classes of devices for accessing information have emerged along with an increased connectivity. In parallel to the proliferation of these devices, new interaction styles have been explored. Among these new styles are virtual reality, mixed reality, 3D interaction, tangible user interfaces, context-aware interfaces and recognition-based interfaces. As a result of this increasing diversity of devices and interaction styles, developers of nextgeneration interfaces experience difficulties such as the lack of appropriate interaction abstractions, the need to create different design variations of a single user interface and the integration of novel hardware. As part of the user interface software research community effort to address these difficulties, the concept of UIDL, which has its foundations in user interface management systems and model-based authoring, has reemerged as a promising approach. UIDLs allow user interface designers to specify a user interface, using high-level constructs, which abstract away implementation details. UIDL specifications can then be automatically or semi automatically converted into concrete user interfaces or user interface implementations. Several UIDLs, mostly using XML as the general language, have been developed in recent years in order to simplify the development of next generation interfaces. However, despite the advancements demonstrated by the UIDL research community (see workshop on developing user interfaces with XML at AVI 2004 [6], and the adoption of this approach in commercial-level applications), many questions regarding the usefulness and effectiveness of UIDLs for next generation user interfaces remain open: What models are required for specifying the dynamic behavior of next generation interfaces that are characterized by continuous, physical and multi-user interactions? How can UIDLs be made understandable and useful to user interface developers from different disciplinary backgrounds? How should UIDL's be evaluated? What UIDL approaches will result in powerful design and run time services? And finally how the result of collaboration between user interface developers and UIDL researchers will affect the UI architectural framework of the next generation of user interfaces?

\section{Workshop Goals}

The first objective of this workshop is to reach a common understanding of the UIDL approach, its potential and shortcomings. The second objective is to identify a set of common challenges that impact emerging and future UIDL research by understanding the perspectives of both user interface developers from different disciplines and UIDL researchers. During the workshop, user-interface developers and UIDL

researchers will work together in teams. Each team will collaborate around an emerging interaction style, leveraging the members various perspectives, with the goal of forming requirements for a UIDL that support this interaction style, and proposing a solution that satisfies these requirements. The strengths and weaknesses of the various solutions will then be compared. Together, this research community will identify common challenges and propose new concepts to solve them. Our last objective is to consider future spaces for UIDL research. This will help the UIDL research community to focus its attention on supporting the $\mathrm{CHI}$ community in its effort to develop the next generation of user interfaces as well as 
recognize opportunities for collaboration.

\section{Participants and Expected Community \\ Interest}

A key goal of this workshop is to foster collaboration between developers of the next generation of user interfaces and user interface software researchers. In particular, the workshop will welcome both participants working in areas such as: virtual and augmented reality, ubiquitous pervasive and handheld interaction, as well as tangible user interfaces, and participants that are or were involved in an effort to develop, use and evaluate UIDLs.

A number of workshops were held in recent years on topics relevant to sub groups of this community: an AVI 2004 workshop on XML-based User Interface Description Languages [6]. A CHI 2005 workshop, The Future of User Interface Design Tools [9] and finally a CHI 2006 workshop, What is the Next Generation of Human Computer Interaction? [3]. We believe that the time is ripe to connect researchers from these areas in order to identify key challenges facing this community at the large, to jointly develop new approaches aimed at solving these challenges and consider future spaces for UIDL research.

\section{Background}

\section{Historical Roots}

In the early 1980's, the concept of a user interface management system (UIMS) was an important focus area for the then-forming user interface software research community [8]. A UIMS allows designers to specify interactive behavior in a high-level user interface description language (UIDL) that abstracts the details of input and output devices. This specification would be automatically translated into an executable program or interpreted at run time to generate a standard implementation of the user interface. The choice of a UIDL model and methods is a key ingredient in the design and implementation of a UIMS. The goal of user interface management systems was not only to simplify the development of user interfaces but also to promote consistency across applications as well as the separation of user interface code from application logic. However, the standardization of user interface elements in the late 1980's on the desktop paradigm made the need for abstractions from input and output devices mostly unnecessary. In addition, user interface developers were seeking control of the user interface look and feel. Thus, although a promising concept, the UIMS approach has been challenged in practice [8]. Subsequently, in the last decade, as a result of the proliferation of new devices and interaction techniques, some of the challenges facing the developers on next generation user interfaces are similar to those that faced GUI developers in the early 1980's. Thus, as part of the user interface software research community effort to address these difficulties, the concept of UIDL reemerged as a promising approach.

\section{Emerging UIDLs}

Several UIDLs have been developed in recent years. Most of them are XML-based. As described in [6] the goals of these emerging UIDLs are:

- To capture the requirements for a user interface as an abstract definition that remains stable across a variety of platforms.

- To enable the creation of a single user interface design for multiple devices and platforms.

- To improve the reusability of a user interface.

- To support evolution, extensibility and adaptability of a user interface. 
- To enable automated generation of user interface code.

To date, we have witnessed an extensive development of UIDLs and frameworks that address the development of user interfaces for multiple platforms, contexts and user profiles. Examples include Plastic User Interfaces [12], UIML[1], XIML[10], UsiXML[5] and the TERESA XML [7]. However, only few UIDLs currently address the development of next generation user interfaces, supporting interaction styles such as virtual reality (VR), mixed reality, ambient intelligence and tangible user interfaces (TUIs): InTML [2] describes VR applications in a platform-independent and toolkitindependent manner. PMIW [4] describes the structure of non-WIMP user interfaces while directly capturing continuous relationships. TUIML [11], draws upon the PMIW approach, and aims at supporting the development of TUIs while explicitly describing continuous and parallel interactions.

In this workshop we aim to harness the potential demonstrated by UIDL research area in supporting the development of multi-platform and multi-modal interfaces to address the challenges facing the developers of the next generation of user interfaces.

\section{References}

[1] Ali, M.F., Perez-Quinones, M.A. and Abrams, M. Building Multi-Platform User Interfaces with UIML. in A., S. and H., J. eds. Multiple User Interfaces, John Wiley and Sons, UK, 2004, 95-116. description language for VR applications The seventh international conference on 3D Web technology, Tempe, Arizona, 2002.
Jacob, R.J.K. What is the Next Generation of Human-Computer Interaction? ACM Press, Workshop abstract, 2006.

Jacob, R.J.K., Deligiannidis, L. and Morrison, S. A Software Model and Specification Language for Non-WIMP User Interfaces ACM Transactions on Computer-Human Interaction, 1999, 1-46.

Limbourg, Q., Vanderdonckt, J., Michotte, B., Bouillon, L. and Lopez, V. UsiXML: a Language Supporting Multi-Path Development of User Interfaces 9th IFIP Working Conf. on Engineering for Human-Computer Interaction jointly with 11th Int. Workshop on Design, Specification, and Verification of Interactive Systems EHCI-

DSVIS'2004 Hamburg, Germany, 2004.

Luyten, K., Abrams, M., Vanderdonckt, J. and Limbourg, Q. Developing User Interfaces with XML: Advances on User Interface Description Languages Advanced Visual Interfaces 2004 Galipoli, Italy, 2004

Mori, G., Paternò, F. and Santoro, C. Design and Development of Multidevice User Interfaces through MultipleLogical Descriptions IEEE

Transactions on Software Engineering 507-520.

Myers, B., Hudson, S.E. and Pausch, R. Past Present, and Future of User Interface Software Tools ACM Transactions on Computer-Human Interaction, 2000, 3-28.

Olsen, D.R. and Klemmer, S.R. The Future of User Interface Design Tools CHI200 5: ACM Conference on Human Factors in Computing Systems, Portland, Oregon, 2005.

10] Puerta, A and Eisenstein, ]. XIML: A Common Representation for Interaction Data IUI2002: Sixth International Conference on Intelligent User Interfaces, 2002.

Shaer, O. and Jacob, R.J.K., A Visual Language for Programming Reality-Based Interaction. in IEEE Symposium on Visual Languages and HumanCentric Computing, Doctoral Consortium, 2006. Thevenin, D., Coutaz, J. and Calvary, G. A Reference Framework for the Development of Plastic User Interfaces. in Multiple User Interfaces, John Wiley \& Sons, , UK, 2004, 29-49. 


\section{Workshop Papers}

\section{A. Frameworks and Paradigms}

Gaelle Calvary, Joel Coutaz, Lionel Balme, Alexandre Demeure, Jean-Sebastien Sottet

Université Joseph Fourier

Clemens Klokmose $\dagger$, Michel Beaudouin-Lafon

$\dagger$ University of Aarhus, $¥$ LRI - Univ. Paris-Sud

Thomas Pederson $\uparrow$, Antonio Piccinno Surie $\dagger$, Carmelo Ardito $\ddagger$, Nicholas Caporusso $\ddagger$, Lars-Erik Janlert $\dagger$

$\nmid$ Umea University, $¥$ Universita degli Studi di Bari

Erik Stolterman $\nmid$,Youn-kyung $\operatorname{Lim} \uparrow$

$\dagger$ Indiana University, $¥$ Korea Advanced Institute of

Science and Technology
The Many Faces of Plastic User Interfaces

From Applications to Ubiquitous Instrumental Interaction

Framing the Next-Generation 'Desktop' using Proximity and HumanPerception

A Model of Interaction

\section{B. Requirements and Considerations for Future UIDLs}

Alexander Behring, Andreas Petter, Felix Flentge, Max Mühlhäuser

TU Darmstadt

Alexandre Demeure, Gaelle Calvary

University of Grenoble
Towards Multi-Level Dialogue Refinement for User Interfaces

Requirements and Models for Next Generation UI Languages 


\section{UIDLs for Multimodal and Ubiquitous Computing}

Mir Ali, Dale Russell, Kibum Kim, Zhuli Xie

Motorola Labs

Cristian Bogdan $\uparrow+$, Hermann Kaindl $\$$, Jürgen Falb

$\dagger$ Royal Institute of Technology (KTH), $\ddagger$ Vienna

University of Technology

Ladry Jeran Francois, Philippe Palanque, Sandra Basnyat, Eric Barboni, David

Navarre

IRIT University Paul Sabatier

Bruno Dumas, Denis Lalanne, Rolf Ingold

University of Fribourg

Jair Leite, Antonio Cosme

UFRN

Fabio Paterno, Carmen Santoro

ISTI-CNR
Dynamic User Interface Creation based on Device Descriptions

Discourse-based Interaction Design for Multi-modal User Interfaces

Dealing with Reliability and Evolvability in Description Techniques for Next Generation User Interfaces

Prototyping Multimodal Interfaces with the SMUIML Modeling Language

XSED: notations to describe status- event ubiquitous computing systems

UIDLs for Ubiquitous Environments 


\section{UIDLs for Task Analysis and Virtual Environments}

\section{Gerrit Meixner, Nancy Thiels}

University of Kaiserslautern

\section{Volker Paelke}

Leibniz Universitaet Hannover, IKG

Chris Raymaekers, Lode Vanacken, Joan De Boeck, Karin Coninx

Hasselt University

Chadwick Wingrave

Virginia Tech

\author{
Tool Support for Task Analysis
}

Spatial Content Models and UIDLs for Mixed Reality Systems

High-Level Descriptions for Multimodal Interaction in Virtual Environments

Chasm: A Tiered Developer-Inspired 3D Interface Representation 


\section{Workshop Participants}

\section{Orit Shaer}

(Organizer)

\section{Robert Jacob}

(Organizer)

\section{Kris Luyten}

(Organizer)

Mark Green

(Organizer)

Mir Ali

Lionel Balme

Michel Beaudouin-Lafon

Alexander Behring

Cristian Bogdan

Gaelle Calvary

Nicholas Caporusso
Tufts University

oshaer (at) cs.tufts.edu

Tufts University

jacob (at) cs.tufts.edu

Hasselt University and transnationale Universiteit Limburg

kris.luyten (at) uhasselt.be

University of Ontario Institute of Technology

mark.green (at) uoit.ca

Motorola Labs

farooqalim (at) gmail.com

Université de Grenoble

lionel.balme (at) imag.fr

Univ. Paris-Sud

mbl (at) lri.fr

TU Darmstadt

behring (at) tk.informatik.tu-darmstadt.de

Royal Institute Of Technology (KTH) cristi (at) nada.kth.se

Université Joseph Fourier, Grenoble gaelle.calvary (at) imag.fr

Università di Bari

ncaporusso (at) gmail.com 
Joelle Coutaz

Alexandre Demeure

Jeff Dicker

Bruno Dumas

Juergen Falb

Ladry Jeran Francois

Clemens Klokmose

Michael Horn

Denis Lalanne

Jair Leite

Youn-kyung Lim
Universite Joseph Fourier, Grenoble

joelle.coutaz (at) imag.fr

Hasselt University

alexandre.demeure (at) uhasselt.be

University of Waterloo, Canada

jadicker (at) cs.uwaterloo.ca

University of Fribourg

bruno.dumas (at) unifr.ch

Vienna University of Technology

falb (at) ict.tuwien.ac.at

\section{IRIT}

dgef213 (at) gmail.com

University of Aarhus

clemens (at) daimi.au.dk

Tufts University

michael.horn (at) tufts.edu

University of Fribourg

denis.lalanne (at) unifr.ch

UFRN

jaircleite (at) gmail.com

KAIST

younlim (at) gmail.com 
Gerrit Meixner

Jeffrey Nichols

(Program Committee)

Volker Paelke

Fabio Paternò

(Program Committee)

Thomas Pederson

Erik Stolterman

Chris Raymaekers

Chadwick Wingrave

Jamie Zigelbaum

(Guest)
University of Kaiserslautern

meixner (at) mv.uni-kl.de

\section{IBM Almaden Research Center}

jwnichols (at) us.ibm.com

Leibniz Universitaet Hannover, IKG

volker.paelke (at) ikg.uni-hannover.de

\section{ISTI-CNR}

fabio.paterno (at) isti.cnr.it

Umea University, Sweden

top (at) cs.umu.se

Indiana University

estolter (at) indiana.edu

Hasselt University

chris.raymaekers (at) uhasselt.be

Virginia Tech

cwingrav (at) vt.edu

MIT Media Lab

zig (at) media.mit.edu 


\section{Discourse-based Interaction Design for Multi-modal User Interfaces}

\section{Cristian Bogdan}

School of Computer Science

and Communication

Royal Institute of Technology

10044 Stockholm, Sweden

cristi@csc.kth.se

and

Institute of Computer Technology Institute of Computer Technology

Vienna University of Technology

A-1040 Vienna, Austria

bogdan@ict.tuwien.ac.at

\author{
A-1040 Vienna, Austria \\ falb@ict.tuwien.ac.at
}

\section{Abstract}

Current user interfaces do not sufficiently utilize

multiple modalities. We developed a new approach to modeling discourse-based interaction design inspired by theories of human communication. From such an interaction design, we envisage to generate a multimodal user interface. This paper presents our approach in the context of mixed-initiative interactions with a (semi-)autonomous robot.

Copyright is held by the author/owner(s).

CHI 2008 Workshop: User Interface Description Languages for Next Generation User Interfaces, April 5 - 10, 2008, Florence, Italy

ACM 1-xxxxxxxxxxxxxxxxxx.

\begin{abstract}
Introduction
In previous work [2] we studied several theories of human communication from various fields to develop an approach for specifying discourse-based interaction design models. These design models are more understandable and possibly easier to build for humans with less technical background than user-interface models. Based on such an approach, we showed in [1] how graphical user interfaces can be rendered from high-level models.

Since the concepts of human communication are applicable to different modalities, we strive for rendering multi-modal interfaces that support mixedinitiative. As a benefit, modelers do not need to care about modality while specifying the interaction design. During rendering the system will suggest one or more modalities that a particular part of an interaction should be performed in. The modeler is still able to influence this decision making. This process should ease the development of multi-modal mixed-initiative interfaces for modelers, since they only have to specify one discourse-based interaction for all modalities.
\end{abstract}

\section{Approach description}

Our approach to multimodal communication consists of two distinct stages: the creation of the interaction 


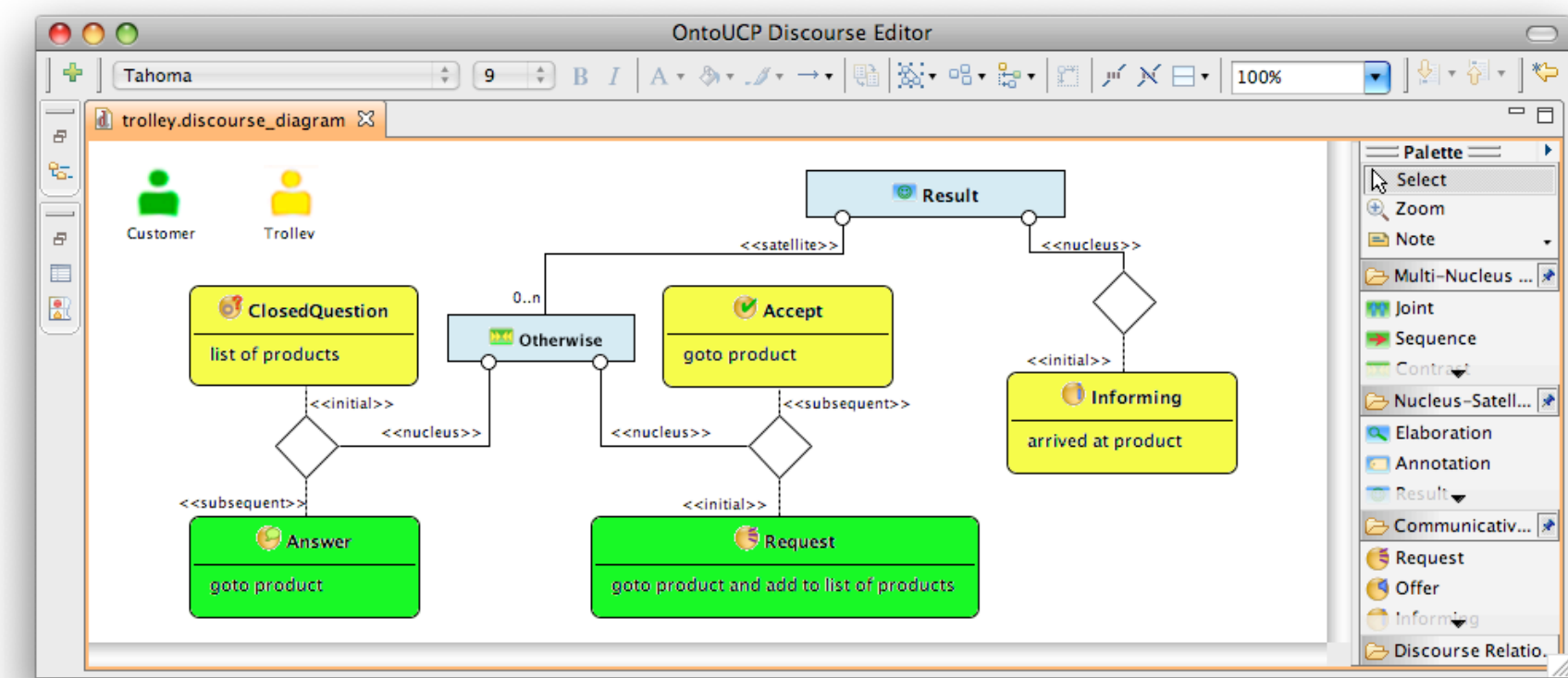

figure 1. The discourse model

model, which is modality-neutral, and the rendering where the modeller and possibly other people can assist the system in improving the interface by placement (spatial or temporal) of components within the constraints of the interaction model, choice of modality, etc. First we focus on the modality-neutral interaction design stage.

We describe our approach to model multimodal communication of humans with (semi-)autonomous robots through an example of a shopping trolley robot that helps the customer to process a predefined shopping list and to find items in a supermarket environment. Through the explanation we emphasize the concepts of human communication that our approach is inspired from. We have modelled (part of) an example interac- tion in figure 1 according to our discourse modelling approach.

A typical scenario covered by the discourse illustrated model goes as follows: First, either the trolley asks the customer to select a product from the shopping list to go to next, or the customer directly requests the trolley to go to yet another product in the supermarket. After specifying the next product, the robot shopping trolley starts moving to the indicated destination together with its assigned customer. When they get to the requested product, the trolley informs the customer about the arrival and removes the product from the shopping list. Our models describe classes of dialogues or scenarios, respectively, in a primarily declarative way. So, this model also includes e.g., that the customer can redirect 
the shopping trolley at any time, through requesting a new product as the current destination.

In the first step of the above scenario, the specification of a product can be accomplished in two different ways, either the trolley asks where to go, or the user requests to go somewhere. The two alternatives are an example of how our modelling framework can accommodate mixed-initiative interaction. We model these alternatives as two adjacency pairs (inspired from Conversation Analysis, details on the human communication concepts and the modelling language can be found in $[1,2])$. These adjacency pairs are grouped together with a rhetorical relation (inspired from Rhetorical Structure Theory (RST)). All our models are, in fact, trees with adjacency pairs as leaves and rhetorical relations as the other nodes. In this case, since the two alternatives are of equal "weight", the adjacency pairs are grouped with an "Otherwise" RST relation, which is meant for such cases.

Indicating a destination from the part of the user is modelled at the bottom-centre, in the form of a "Request" communicative act inspired from Speech Act Theory. Communicative acts offer us an abstraction that is graphical-toolkit-neutral and also modalityneutral. Adjacent to the request, there is an "Accept" communicative act with which the machine confirms the new destination. The left side of the model offers the collected destinations for the user to choose from. This is modelled as a "Closed Question" communicative act, to which the user can respond, by way of the adjacent "Answer" communicative act, to choose from a defined list of possibilities. This list of possibilities is called propositional content in Speech Act Theory, and in our approach it is provided and refreshed by the application logic of the robot trolley. The "Closed Question" also helps the user to keep updated on what the shopping items are that were already added to the shopping list but not yet processed.

If there is no further user interaction and the robot reaches the destination currently specified, it informs the user about the status via the "Informing" communicative act at the right of our model, and the destination is removed from the shopping list by the robot's application logic. Since this is the main result of the interaction, the "Informing" is linked to the remainder of the dialogue model through a "Result" RST relation.

\section{Multimodal Communication with a Robot according to this Model}

Now let us focus on the rendering stage where the communication platform software will have to deal with modalities for expressing and receiving communicative acts. It is designed to do so based on heuristics, but the modeller and possibly other people may assist in choosing one or multiple modalities for improving the interface. Our robot trolley is designed to support three communication modalities and their combination: graphical interaction through a touch screen, speech input/output and movement.

Since the "Request goto product" communicative act is modelled to give the application logic data of a certain type (let's call it destination) and a speech input of type destination is available from the speech recognition, the render engine will recognize that the "Request" can be rendered in speech input. While assisting the rendering process, the modeller can decide that the request can also be done via the touch screen, in which case e.g., a widget providing alphabetical search for destinations 
can be rendered. Furthermore, our communication platform software can decide at runtime to fall back to the graphical input in a very noisy environment. In the case of accepting a "Goto Request", the trolley will utter the Accept communicative act in e.g., speech, since using the same modality for the adjacent communicative act improves clarity and answers the user's expectation.

The render engine will, in principle, render the "Closed Question" with the shopping list items only on the touch screen, as the speech medium is an expensive resource for a list. However, if desired at the rendering stage, speech could also be used in this case. This could be based e.g., on the level of ambient sound i.e., if the user appears to be alone in the shop, there is more "rendering space". And, maybe after periods of no communication with the user although sensed to be in the robot's proximity, the "Closed Question" can be uttered in speech as a suggestion. The user can interrupt the utterance via a predetermined speech utterance, to indicate that she chose the last destination uttered by the robot speech synthesis. In previous work, we have used the rendering space as a constraint for model rendering in GUI interfaces, but as exemplified here, a similar temporal constraint can be used for the speech modality.

\section{Discussion and Conclusion}

Our approach can be regarded as a very-high-level user interface definition language, or more precisely an interaction design language. We envisage that from the communicative acts, rhetorical relations and conversation analysis patterns it employs, a decent multi-modal interface can be generated. If a pre- rendering stage is added, our render engine will get even more guidelines for its runtime heuristics, resulting in higher-quality interfaces.

We have also shown that although our interaction models are modality-neutral, the modality can be inferred from the model in multiple ways: from data types involved, from the availability of widgets for the respective modality, from quasi-spatial constraints in the modality, and not the least from the "importance" of a certain communicative act as conveyed by the rhetorical structure of the discourse. If such inferences do not suffice, based on the interaction model, our system will be able to guide the modelers and designers to specify the modality of certain communicative acts.

\section{Acknowledgements}

We would like to thank Helge Hüttenrauch and Alexander Szep for their reviews and valuable comments.

\section{References}

[1] Bogdan, C., Falb, J., Kaindl H., Kavaldjian S., Popp R., Horacek H., Arnautovic E. and Szep A. Generating an Abstract User Interface from a Discourse Model Inspired by Human Communication. In Proc. 41th Annual Hawaii Int'l Conference on System Sciences (HICSS-41), IEEE Computer Society Press (2008).

[2] Falb J., Kaindl H., Horacek H., Bogdan C., Popp R., and Arnautovic $\mathrm{E}$. A discourse model for interaction design based on theories of human communication. In Ext. Abstracts CHI 2006, ACM Press (2006), 754-759. 